\title{
Optimization of adaptive feedback control for ultrafast semiconductor spectroscopy
}

\author{
Jens Kunde, Benedict Baumann, Sebastian Arlt, and François Morier-Genoud \\ Ultrafast Laser Physics, Institute of Quantum Electronics, Swiss Federal Institute of Technology, ETH Hoenggerberg \\ HPT, CH-8093 Zurich, Switzerland \\ Uwe Siegner \\ Physikalisch-Technische Bundesanstalt, Bundesallee 100, D-38116 Braunschweig, Germany \\ Ursula Keller \\ Ultrafast Laser Physics, Institute of Quantum Electronics, Swiss Federal Institute of Technology, ETH Hoenggerberg
} HPT, CH-8093 Zurich, Switzerland

Manuscript received August 17, 2000; revised manuscript received December 5, 2000.

\begin{abstract}
We present an experimental study of the control of ultrafast semiconductor nonlinearities by adaptive feedback optical pulse shaping. In the feedback loop, an evolutionary algorithm directs the modulation of the spectral phase of 20-fs laser pulses. In this way, control is achieved over the broadband semiconductor continuum nonlinearity as measured in differential transmission experiments. Design guidelines are given for the implementation of the evolutionary algorithm. Our results demonstrate that a feedback loop with a carefully designed algorithm can serve as a new, sensitive tool in ultrafast semiconductor spectroscopy. Moreover, an optimized feedback loop allows for the substantial enhancement of ultrafast semiconductor nonlinearities. (C) 2001 Optical Society of America
\end{abstract}

OCIS codes: $320.5540,320.7130,320.7150$.

\section{INTRODUCTION}

In recent years adaptive feedback optical pulse shaping has found more and more applications in ultrafast optics. $^{1-13}$ This scheme uses a computer-based algorithm that iteratively changes the shape of an optical pulse to reach a preset goal. It has been demonstrated that this self-learning technique offers a number of attractive features. First, this scheme can work successfully if the optical field that optimizes a given process is not known a priori. Moreover, this technique can work with partially uncharacterized input pulses, can compensate for slow drifts in the experiment, and is robust against experimental noise. Exploiting these features, adaptive feedback optical pulse shaping has been used in various fields, such as optical pulse compression, ${ }^{1-5}$ the synthesis of predefined optical pulse shapes, ${ }^{6}$ pulse propagation in optical fibers,${ }^{7}$ high-harmonic generation, ${ }^{8}$ the control of molecules, ${ }^{9-12}$ and the shaping of the quantum wave function of atoms. ${ }^{13}$

In a recent letter, ${ }^{14}$ we demonstrated use of adaptive feedback optical pulse shaping for the control of ultrafast semiconductor nonlinearities. These nonlinearities are an ideal arena for adaptive feedback schemes because, after many years of extensive research, ${ }^{15}$ the complex physics involved $^{15,16}$ still makes it difficult to reliably predict the exact optical pulse shape that produces a desired nonlinear response. It has been shown ${ }^{14}$ that adaptive feedback control gives insight into light-semiconductor interaction and can significantly enhance ultrafast semiconductor nonlinearities. An evolutionary algorithm, more precisely an evolution strategy (ES), ${ }^{17}$ was used to direct the shaping of the spectral phase of the optical pulses within a feedback loop. In this paper we describe our implementation of the ES. We show that the convergence speed and the reliability with which this algorithm finds an optimum solution strongly depend on the details of its implementation. In particular, we discuss the influence of two issues. First, we investigate the role of the representation of the spectral phase, i.e., the way in which the spectral phase is parameterized. Second, the way the ES changes the parameters of the spectral phase also influences the performance of the feedback loop. In particular, we compare different crossover operators. ${ }^{17}$ Design guidelines are given for the optimization of the ES. Our results demonstrate that, with a carefully designed algorithm, adaptive feedback optical pulse shaping can serve as a new, sensitive tool in ultrafast semiconductor spectroscopy. Even if the physics involved is too complex to predict an optimum phase $a$ priori, the results of the optimization a posteriori give new insight into light-semiconductor interaction. Moreover, such a feedback loop allows one to enhance ultrafast semiconductor nonlinearities by almost a factor of 4 , which makes the scheme interesting for the optimization of ultrafast all-optical switching devices.

The design guidelines given for the optimization of the ES are not restricted to applications in semiconductor spectroscopy but should also have an effect on other fields 
in which adaptive feedback optical pulse shaping is used. ${ }^{1-13}$

The paper is organized as follows. In Section 2 we present our experimental setup and discuss the methods used for pulse characterization. The implementation of the ES and its optimization for adaptive feedback optical pulse shaping is presented in Section 3. In Subsection 3.A we discuss the basics of ESs and their application to adaptive feedback optical pulse shaping. The influence of the different parameters that describe the spectral phase is the subject of Subsection 3.B. Guidelines are given for an adequate representation of the spectral phase. In Subsection 3.C we experimentally compare the performance of the feedback loop for different crossover operators. We determine the crossover operators that give the best results in terms of convergence speed and reliability with which the ES finds an optimum solution. This optimized ES is used for the control of ultrafast semiconductor nonlinearities in Section 4. In Subsection 4.A we discuss the optimization of the spectrally integrated (SI) differential transmission (DT). From the shape of the optimum phase an intuitive picture of the photon energy dependence of carrier relaxation in the thermalization regime is obtained. We compare our experimental data with results from a semiquantitative model. Subsection 4.B deals with the optimization of the DT in narrow spectral windows. It is demonstrated that this DT can be enhanced by a factor of approximately 4 . We finally present our conclusions in Section 5 .

\section{EXPERIMENTAL SETUP}

Figure 1(a) shows our experimental setup, which consists of three main parts: a computer-based ES, a programmable pulse shaper, and a pump-probe setup for the acquisition of SI DT data and DT spectra. The DT measurements provide the feedback signal for the ES, which optimizes the DT by changing the settings of the pulse shaper and the delay between the pump and the probe pulses. The operation of the ES is described in Section 3.

In the pump-probe setup, pulses from a 20-fs Ti:sapphire laser are used for standard noncollinear DT measurements with linearly cross-polarized pump and probe pulses. We studied a 1 - $\mu \mathrm{m}$-thick $\mathrm{Al}_{0.06} \mathrm{Ga}_{0.94} \mathrm{As}$ bulk semiconductor sample whose GaAs substrate was removed by chemical wet etching to allow for transmission experiments. The sample was glued onto a sapphire substrate, and a broadband antireflection coating was deposited on its air interface. Figure 1(b) shows that mainly continuum transitions are excited. The excitation carrier density is $N_{\text {exc }} \approx 3 \times 10^{17} \mathrm{~cm}^{-3}$. All experiments were done at room temperature. For the acquisition of the DT, we use chopping techniques and lock-in detection. A lowdispersive mechanical high-frequency chopper allows for DT measurements with high sensitivity. ${ }^{18}$ The programmable pulse shaper is positioned in front of the pumpprobe beam splitter. Therefore both pulses, pump and probe, experience the same modulation. After passing dispersion-balanced paths, the pump and probe pulses exhibit the same spectral amplitude and phase at the sample. In the pulse shaper, the spectral components are filtered by a programmable liquid-crystal spatial light modulator (SLM) placed at the Fourier plane of a 4-f system. ${ }^{19,20}$ The 4 - $f$ system is composed of a pair of reflective gratings with 600 grooves/mm and a pair of cylindrical lenses. The SLM consists of two liquid-crystal masks (SLM-256-NIR from Cambridge Research and Instrumentation). This arrangement allows for the independent control of the spectral amplitude and the spectral phase at 128 pixels. $^{21}$ Unless otherwise mentioned, we used the pulse shaper for manipulation of the spectral phase only without modification of the spectral amplitude.

When using such a pulse shaper for DT spectroscopy, we must carefully check for sources of possible artifacts. It is known, for example, that frequency-domain pulse shaping with spatially patterned masks leads to spacetime coupling. ${ }^{22,23}$ Moreover, the pixelation of the mask and the gaps between adjacent pixels of the SLM may lead to unwanted side effects. ${ }^{20,24}$ We carefully verified by calculations as well as by experiments that, for our pulse-shaping setup, these artifacts do not distort our experimental results.

For the characterization of the spectral phase we used two independent methods. As a first method, we use second-harmonic generation (SHG) frequency-resolved optical gating (FROG). ${ }^{25}$ Recently it has been demonstrated that FROG reliably recovers even the spectral phase of complex SLM-shaped waveforms. ${ }^{26}$ Alternatively, the spectral phase can also be obtained from the

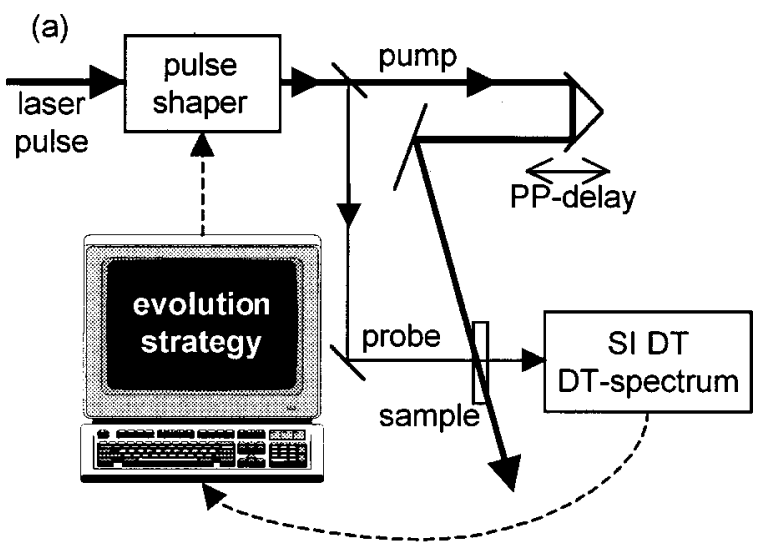

(b)

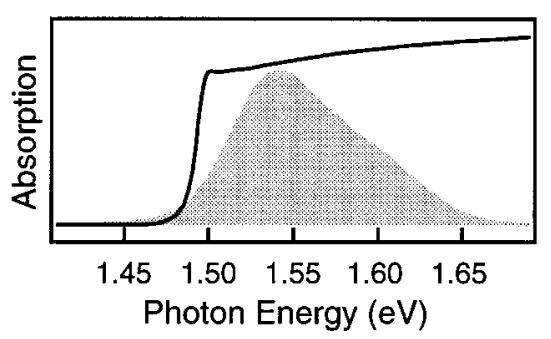

Fig. 1. (a) Experimental setup for adaptive feedback control of the SI DT and the DT in narrow spectral windows. Solid lines indicate the optical beam path. The dashed lines illustrate how the feedback loop is closed between the experiment and the pulse shaper. The ES also directs the pump-probe (PP) delay. For pulse compression experiments, the semiconductor sample is replaced with a $10-\mu \mathrm{m}$-thick $\beta$ barium borate crystal, and the timeintegrated second-harmonic generation signal of the pump or the probe pulse serves as merit function. (b) The absorption spectrum of the $\mathrm{Al}_{0.06} \mathrm{Ga}_{0.94} \mathrm{As}$ sample (solid curve) and the excitation pulse spectrum (shaded area) are shown. 
voltage settings of the SLM. This approach requires a calibration of the voltage-phase relation of the liquidcrystal mask, which is otherwise not mandatory when one uses adaptive feedback optical pulse shaping. ${ }^{2}$ However, in Subsection 3.B we show that such a calibration also offers certain advantages for use of adaptive schemes. With this calibration, the spectral phase modulation $\varphi_{\text {SLM }}$ induced by the SLM is obtained. To get the actual spectral phase $\varphi$ at the position of the sample, $\varphi_{\text {SLM }}$ has to be corrected for the phase $\varphi_{\text {setup }}$ of all other optical elements in the experimental setup, i.e., $\varphi=\varphi_{\text {setup }}+\varphi_{\text {SLM }}$. For the measurement of $\varphi_{\text {setup }}$, adaptive feedback optical pulse shaping can also be used. Following Refs. 1 and 2, we replace the semiconductor sample with a thin SHG crystal ( $10-\mu \mathrm{m} \beta$ barium borate). Then we use the adaptive feedback loop to maximize the time-integrated SHG signal. According to Ref. 27, this maximization corresponds to a flat temporal phase, i.e., to a compressed pulse. For a symmetric pulse spectrum, a flat temporal phase corresponds to a flat spectral phase, i.e., the optimized phase $\varphi_{\text {SHG,max }}$ compensates for the phase of the setup: $\varphi_{\text {setup }}=-\varphi_{\text {SHG, } \max }$. For asymmetric pulse spectra, a flat temporal phase does not exactly correspond to a flat spectral phase. We numerically checked that, in our experiments, the resulting error in the spectral phase characterization is negligibly small. Moreover, as an additional check, we used the spectral amplitude manipulation capability of the pulse shaper to obtain a spectrum that is symmetric in the frequency domain. Pulse compression experiments with both slightly asymmetric and symmetric spectra yield the same spectral phase $\varphi_{\text {SHG,max }}$ within experimental accuracy. The spectral phase data obtained from shaper settings and pulse compression experiments show an excellent agreement with the FROG data. With both methods we also verified that the pump and probe pulses exhibit the same spectral phase at the sample.

\section{IMPLEMENTATION OF THE EVOLUTION STRATEGY}

Evolution strategies have been described in detail, e.g., in Refs. 17 and 28 . In this section we briefly recall the basic ideas of ES with emphasis on our particular implementation. In many experimental tests, we optimized the ES for our application. These test runs were performed for optical pulse compression as well as for the optimization of different parameters of the SI DT and the DT spectrum. The data presented in this section are mainly pulse compression data because the reliability of the achieved results can be easily checked with FROG measurements, which show whether a bandwidth-limited pulse has been obtained. We carefully checked that the guidelines that are developed for the optimization of the ES qualitatively hold for pulse compression and for the optimization of ultrafast semiconductor nonlinearities as measured in DT. As an example, data for both control problems are presented in Subsection 3.C. The optimized ES is used as a sensitive tool in ultrafast semiconductor spectroscopy in Section 4.

\section{A. Basic Algorithm}

The ES is a global optimization procedure that is motivated by biological evolution. We call the parameters that are changed by the algorithm genes, and a combination of the total number $N$ of genes is referred to as an individual. The algorithm works simultaneously on a number of individuals called population. In one cycle of the algorithm, an old population is replaced by a new one by use of the genetic operations of recombination, mutation, and selection. The different steps of this loop are explained in the following.

First we generate 100 individuals. This initialization uses random numbers, which leads to a uniform distribution in the $N$-dimensional search space. The individuals are evaluated, i.e., the merit function (for example, the time-integrated SHG signal) is determined experimentally. The 15 best or fittest individuals build the starting population for the feedback loop.

(i) Recombination. From the population, two individuals are chosen randomly. These individuals, the parents, produce one offspring by interchanging genes. The way this interchange takes place influences the overall performance of the feedback loop. A comparison of different types of recombination, also referred to as different crossover operators, is presented in Subsection 3.C. The recombination process is repeated 100 times, i.e., 100 offspring are generated from the 15 members of the previous generation.

(ii) Mutation. Each of the 100 offspring is now mutated, i.e., the values $x_{i}$ (also called object variables) of the $N$ genes are changed. The magnitude of these changes is proportional to the mutation step lengths $\sigma_{i}$, which are also referred to as strategy variables. All variables $x_{i}$ and $\sigma_{i}$ are changed by the feedback loop. For the mutation, we follow the approaches described in Refs. 17 and 28.

(iii) Selection. The 100 individuals are now evaluated. The 15 individuals with the highest merit function build the population of the next generation. ${ }^{29}$ When presenting data achieved with the ES, we chose to restrict ourselves to the average over these 15 individuals throughout this paper. With this selection scheme, none of the individuals of the old population are carried on to the new one. Alternatively, in an elitist version of ES, one can put the best individuals of the old generation into the new one without applying recombination or mutation. ${ }^{17} \mathrm{Un}$ der laboratory conditions, i.e., in the presence of noise and drift, we found this scheme to be unfavorable.

The loop with steps (i-iii) continues until terminated by the user. Because of the self-adaptive step length control, optimization in such an ES is a two-stage process as illustrated in Fig. 2. For an adaptive pulse compression experiment, the phase differences between the 128 pixels of the SLM were optimized. Figure 2 shows the average $\sigma_{\text {av }}$ of all mutation step lengths and the root-mean-square deviation $\Delta \varphi_{\text {rms }}$ between the current spectral phase and the final optimized phase evaluated at the 128 pixels. The average mutation step length first increases, leading to a fast convergence of the spectral phase toward the optimum. After the spectral phase has reached a point near the optimum, the average mutation step length de- 


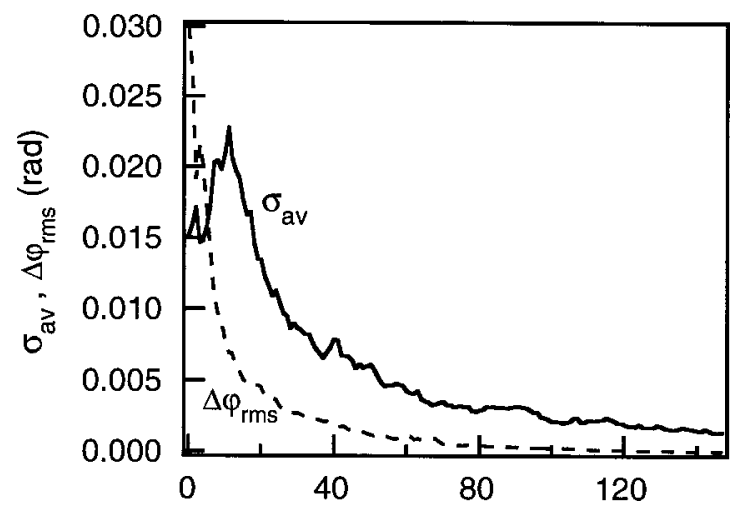

Fig. 2. Average mutation step length $\sigma_{\mathrm{av}}$ and root-mean-square phase deviation $\Delta \varphi_{\text {rms }}$ for a pulse compression experiment.

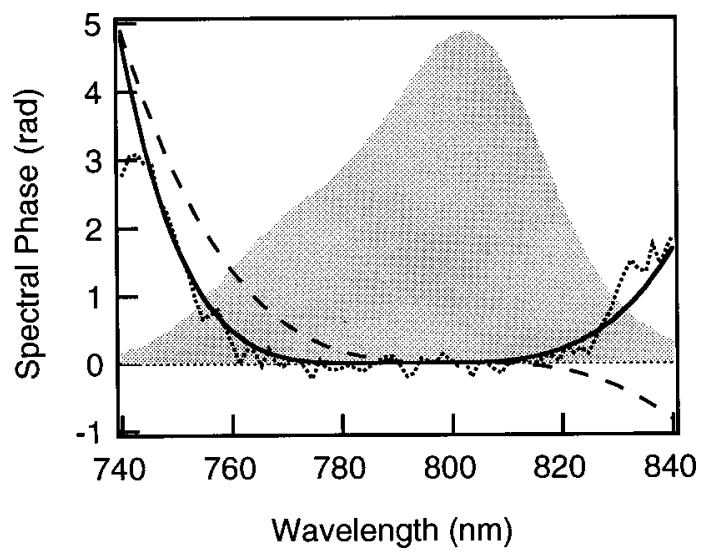

Fig. 3. Optimized phase shapes for pulse compression experiments for a chirp expansion up to third-order dispersion (dashed curve), up to sixth-order dispersion (solid curve), and for the optimization of the spectral phase differences between all pixels independently (dotted curve). The shaded area is the excitation pulse spectrum.

creases, so that the region of the search space close to the optimum can be explored more accurately.

\section{B. Choosing and Initializing the Genes}

For pulse compression, the ES changes only the spectral phase of the optical pulses. For DT optimization, the ES also changes the time delay between the pump and the probe pulse (because the DT depends on both), the spectral phase of the pump and the probe pulse, and the delay between both pulses. ${ }^{30}$ For both optimization procedures, the ES needs parameters, or object variables, that describe the spectral phase. Two approaches are used mainly in adaptive feedback optical pulse shaping., ${ }^{1,2}$ First, one can expand the spectral phase into a Taylor series around a center angular frequency $\omega_{0}$ :

$$
\begin{aligned}
\varphi(\omega)= & \frac{1}{2} d^{2} \varphi /\left.\mathrm{d} \omega^{2}\right|_{\omega=\omega_{0}}\left(\omega-\omega_{0}\right)^{2} \\
& +\frac{1}{6} \mathrm{~d}^{3} \varphi /\left.\mathrm{d} \omega^{3}\right|_{\omega=\omega_{0}}\left(\omega-\omega_{0}\right)^{3}+. . \\
= & \operatorname{GDD}\left(\omega-\omega_{0}\right)^{2}+\operatorname{TOD}\left(\omega-\omega_{0}\right)^{3}+. .
\end{aligned}
$$

In such a chirp expansion, the phase is characterized by the group-delay dispersion GDD, the third-order dispersion TOD, and so on. We omitted the constant and the linear phase term because these terms do not influence the results of our experiments. A second approach optimizes the spectral phases at the 128 pixels of the SLM independently of each other.

Naturally, the question arises as to which approach one should follow and, if using the chirp expansion, up to which order this expansion should be carried out. On the one hand, one would like to keep the description of the spectral phase as general as possible; on the other hand the optimization in a higher-dimensional search space is more time-consuming. To answer the above question for a given optimization problem, we first performed an optimization of all pixels independently of each other. Then, in additional runs of the feedback loop, we experimentally checked as to which order a chirp expansion has to be carried out to yield a good fit to the optimum phase of the all-pixel optimization and to yield an equally high merit function. Figure 3 shows the results of this procedure for the example of a pulse compression experiment. Clearly, a chirp expansion up to third-order dispersion gives a poor fit to the phase determined by the all-pixel optimization. In contrast, a chirp expansion up to sixth-order dispersion yields a good fit. This behavior is also reflected by the merit function. Therefore, in this particular experimental situation, a chirp expansion up to sixth-order dispersion is a good compromise between accuracy and convergence speed. For optimization problems that require more complex phase shapes, a chirp expansion may be inadequate as we show in Subsection 4.B. In such cases one should stay with the independent optimization of the spectral phases at each pixel of the SLM.

When one chooses this full optimization, there are still various possibilities for the exact selection of the object variables. For example, one can use the voltage settings at the 128 pixels of the liquid-crystal SLM as object variables. ${ }^{2,11,27}$ This scheme has the advantage that calibration of the voltage-phase relation of the liquid-crystal SLM is not necessary. For our purposes, however, we found that using the real physical quantity, i.e., the spectral phase, leads to a faster convergence of the feedback loop. This is probably because usage of the spectral phase allows for a uniform initialization in the physically relevant search space. According to Ref. 17, such a uniform initialization increases the chance of a fast convergence to the global optimum. We note that an initialization that is uniform in the voltage space is nonuniform or biased in the phase space because the phase depends strongly nonlinearly on the applied voltage for a liquidcrystal SLM. ${ }^{24}$ Moreover, we found that using the phase differences between adjacent pixels as object variables leads to a faster convergence than just using the phases at the pixels. Therefore we choose the phase differences between the 128 pixels as object variables whenever the control problems are so complex that all pixels have to be optimized independently.

\section{Choosing the Crossover Operator}

For recombination, a specific crossover operator has to be chosen that determines how the genes of two randomly 
chosen parents are intermixed to produce an offspring. Although ES recombination is not as important as mutation for convergence, it is still a relevant operation, e.g., to guarantee that the self-adaption of the mutation step lengths works properly. ${ }^{17}$ In this subsection we compare the performance of the ES in terms of convergence speed and reliability of the results for different crossover operators. Figure 4 explains how these operators work. For the offspring produced by discrete crossover, the object variable of each gene is chosen randomly from parent 1 or parent 2, i.e., the object variables of both parents are intermixed randomly. For a one-point crossover, one position in the gene chain is chosen randomly. Then all object variables up to this position are taken from parent 1 , the others from parent 2 . The arithmetic average of the object variables of both parents is taken for the offspring in the intermediary crossover. In our experiments, these three crossover operators are used for the object variables, whereas the intermediary crossover has always been used for the mutation step lengths. In addition, we tested an ES without the recombination procedure. In this variant, we simply replicated randomly chosen individuals from the old population without changing the object or strategy variables.

First we tested the different ES variants in pulse compression experiments. As genes, the spectral phase terms of the chirp expansion up to the sixth-order dispersion, i.e., five genes, were used in one experimental series. In a different series, the phase differences between the pixels of the SLM are optimized independently. For both series, the ES using the intermediary crossover operator for the object variables turns out to be the least reliable one. It shows the tendency of early stagnation with a nonoptimized phase shape, and strongly varying results were achieved for different runs. The three other types of ES perform well in terms of reliability. In each run, the phase shape that maximizes the merit function is found. Therefore we reject the ES that uses intermediary crossover for the object variables and compare the other ES in terms of convergence speed. Figure 5 shows the convergence of the merit function for the three ES's. Five different runs were averaged for all curves. For pulse compression by use of the phase parameters from a chirp expansion, Fig. 5(a) shows that the ES with the onepoint crossover performs slightly better than the one with the discrete crossover operator. Both ESs show a significantly better convergence than the ES that uses no recombination. For the latter, the monitoring of the mutation step lengths reveals that this slow convergence is closely related to a bad self-adaptive alignment of the mutation step lengths as expected for the ES without recombination. ${ }^{17}$ As shown in Fig. 5(b) for pulse compression by use of the parameters of the phase differences between all pixels, the ES using discrete crossover performs significantly better than the one-point crossover ES. Again, use of no recombination results in the poorest convergence speed.

In summary, use of discrete or one-point crossover in ES guarantees a fast and reliable convergence of the pulse compression loop. Which crossover operator should be chosen depends on the parameters that are selected to describe the spectral phase. With an increasing

\begin{tabular}{|l|l|l|l|l|l|}
\cline { 2 - 5 } parent 1 & 2.1 & 5.9 & 7.2 & 4.5 & 1.5 \\
\hline
\end{tabular}
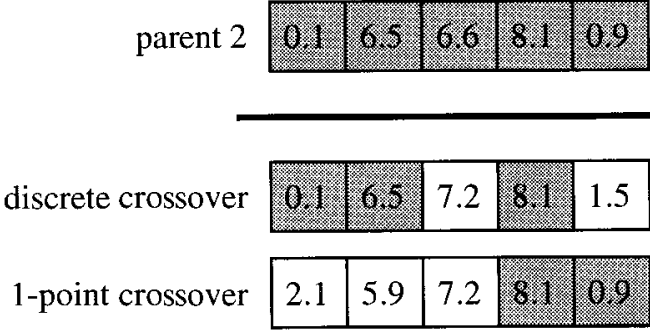

intermediary crossover \begin{tabular}{|l|l|l|l|l|l|l}
\hline 1.1 & 6.2 & 6.9 & 6.3 & 1.2 \\
\hline
\end{tabular}

Fig. 4. Comparison of three different crossover operators that can be used to produce one offspring from parents 1 and 2 .
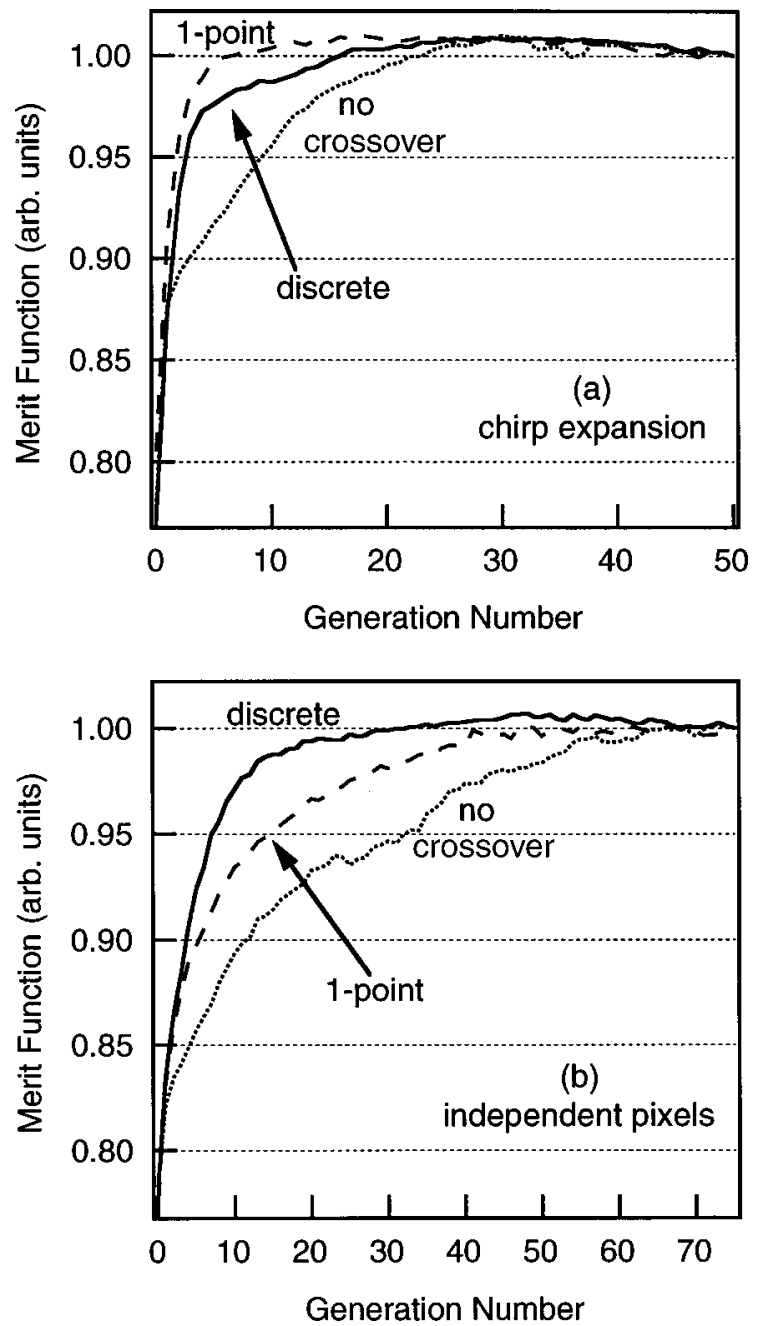

Fig. 5. Pulse compression experiments. Comparison of the convergence speed with a one-point crossover (dashed curves), discrete crossover (solid curves), and without crossover operator (dotted curves). (a) A chirp expansion up to sixth-order dispersion was used, and (b) the spectral phase differences between all pixels were optimized independently.

number of genes, one-point crossover becomes less favorable. In additional experiments we carefully checked that the same results hold for the optimization of the DT of ultrafast semiconductor nonlinearities. As an ex- 
ample, Fig. 6 compares the convergence of the feedback loop for three different crossover operators for DT maximization in a narrow spectral window around $1.625 \mathrm{eV}$. This control problem, which is discussed in detail in Subsection 4.B, requires the independent optimization of all pixels. As shown in Fig. 6, the ES with the discrete crossover operator converges faster than the one with the one-point crossover, and when no recombination is used, it results in the slowest convergence. These results are identical to the results for pulse compression experiments that use the independent optimization of all pixels [compare Fig. 5(b) and Fig. (6)].

The crossover operators were chosen as representative examples for the comparison of different ESs in terms of convergence speed and reliability of the results in this subsection. A further optimization might be possible if one uses other crossover operators, similar to some approaches in genetic algorithms. ${ }^{28,31}$ For our purposes, the performed optimization of the ES gives us a powerful tool for ultrafast semiconductor spectroscopy as demonstrated in Section 4. We mention that an optimization of

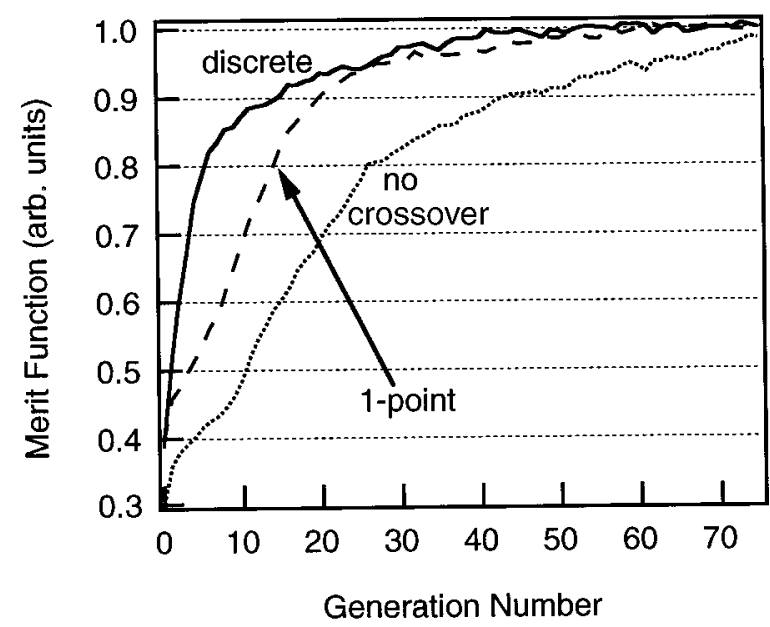

Fig. 6. DT experiments. Comparison of the convergence speed with a one-point crossover (dashed curve), discrete crossover (solid curve), and without a crossover operator (dotted curve) for the maximization of the DT at $1.625 \mathrm{eV}$. The spectral phase differences between all pixels were optimized independently.

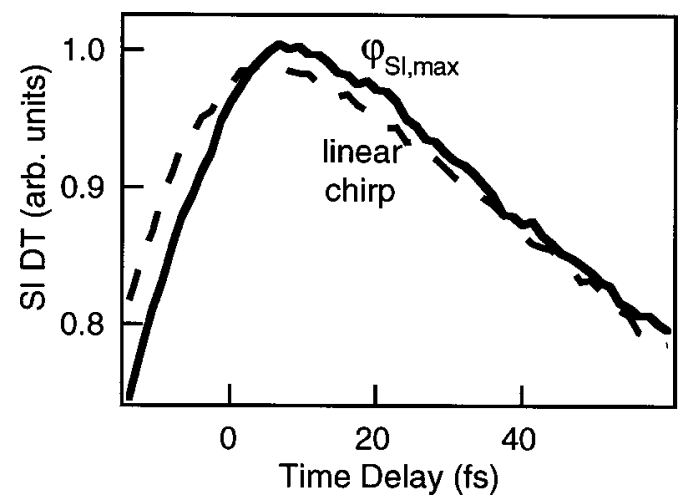

Fig. 7. SI DT for the optimized phase $\varphi_{\text {SI, max }}$ and for the optimization of only the slope of a linear chirp. Temperature is $300 \mathrm{~K} ; N_{\mathrm{exc}} \approx 3 \times 10^{17} \mathrm{~cm}^{-3}$. the algorithm for a different class of problems may result in a different implementation of the ES.

\section{CONTROLLING ULTRAFAST SEMICONDUCTOR NONLINEARITIES}

A. Spectrally Integrated Differential Transmission

In a first experiment, the feedback loop maximizes the SI DT by changing the spectral phases of the pump and probe pulses as well as the pump-probe delay. We used a chirp expansion of the spectral phase up to sixth-order dispersion because it gives a sufficient accuracy for the optimized phase shape (see Subsection 3.B). In Ref. 30 the influence of a linear chirp on the SI DT was investigated. In a chirped pulse, the different frequency components, which make up the broadband pulse, are distributed over the temporal pulse profile. If the highfrequency components are in the leading edge of the pulse and the low-frequency components arrive later, the pulse is downchirped. If this sequence is reversed, the pulse exhibits an upchirp. A linear chirp corresponds to a group delay of $\mathrm{d} \varphi / \mathrm{d} \omega$ that varies linearly with frequency, i.e., a purely quadratic spectral phase. In Ref. 30 it was demonstrated that linear downchirp enhances the SI DT as compared with linear upchirp, or no chirp. However, it could not be decided whether nonlinear downchirp, i.e., a more complex spectral phase, leads to an even larger enhancement. This question is answered by adaptive feedback optical pulse-shaping experiments. Figure 7 shows SI DT transients for the optimized phase $\varphi_{\text {SI,max }}$ as well as for an optimized linear chirp. Note that the optimized phase $\varphi_{\text {SI,max }}$ leads to a SI DT signal that is enhanced by only slightly more than $1 \%$ as compared with the SI DT for linear chirp. Because the SI DT signal ${ }^{32}$ is of the order of a few percent, this corresponds to an absolute difference in the $10^{-4}$ range. Figure 7 shows that spectral phases that lead to these small differences can be easily distinguished after the algorithm is optimized as described in Section 3. In the following, we discuss that this capability allows one to obtain a deeper insight into the underlying carrier dynamics.

Figure 8(a) shows the optimized spectral phases for different experimental runs. The average over several optimization runs yields the optimum phase $\varphi_{\text {SI, max }}$. Although different in detail, all phases correspond to downchirp that differs significantly from a purely linear chirp. This is also visualized by the group delay shown in Fig. 8(b). In a recent letter, ${ }^{14}$ we explained how this behavior correlates to the underlying carrier thermalization dynamics. In essence, the shape of the optimum group delay $\mathrm{d} \varphi_{\mathrm{SI} \text {, max }} / \mathrm{d} \omega$ [see Fig. $8(\mathrm{~b})$ ] is adapted to the changes of the DT spectrum. During carrier thermalization, an almost symmetrical spectral hole evolves into a Fermi-Dirac distribution. ${ }^{15}$ The center frequency of the optimum pulse shape and the maximum of the DT spectrum both shift in the same way to lower energies with time. Therefore the pulse shape that maximizes the SI DT gives an intuitive picture of the complex energy dependence of carrier relaxation in the thermalization regime. A slow variation of the group delay with energy corresponds to a fast shift of the DT spectrum. Hence the optimum group delay shown in Fig. 8(b) indicates that 

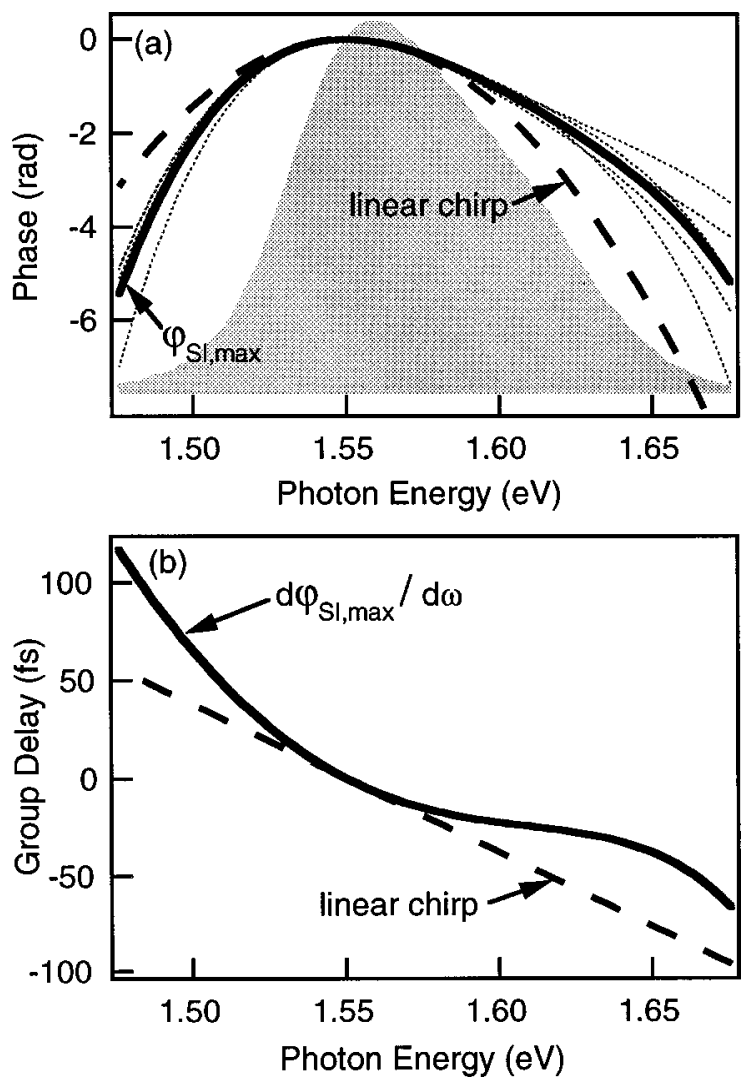

Fig. 8. (a) Average of several optimization runs (dotted curves) yields the optimum phase $\varphi_{\text {SI, max }}$ (solid curve), which maximizes the SI DT. Shown for comparison is the phase corresponding to a linear chirp (dashed curve). The shaded area is the excitation pulse spectrum. Temperature is $300 \mathrm{~K} ; N_{\mathrm{exc}} \approx 3 \times 10^{17} \mathrm{~cm}^{-3}$. (b) Group delay $(\mathrm{d} \varphi / \mathrm{d} \omega)$ obtained from $\varphi_{\mathrm{SI} \text {, max }}$ (solid curve) as compared with a linear chirp (dashed curve).

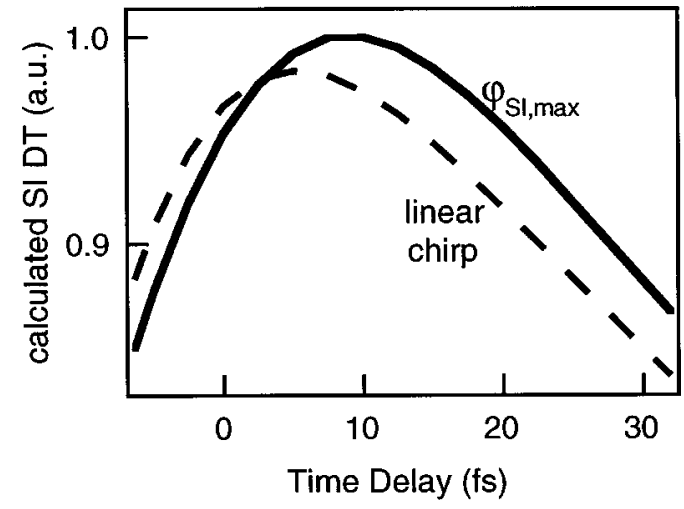

Fig. 9. Calculated SI DT in arbitrary units for the optimized phase $\varphi_{\mathrm{SI} \text { max }}$ and for the optimization of only the slope of a linear chirp.

relaxation is faster at higher energies. To verify these qualitative considerations, we performed numerical simulations using the semiquantitative model introduced in Ref. 30. This model can account for the general trends of chirp influence on the SI DT by calculating how DT contributions from different pump energy components are read out by different probe energy components. Figure 9 shows the calculated SI DT for a linearly downchirped pulse and for the experimentally determined optimum pulse shape. The comparison of experimental and calcu- lated data (Figs. 7 and 9) shows good qualitative agreement. The model also predicts a slightly higher SI DT for the optimum spectral phase as compared with linear downchirp. In the model, it is assumed that a thermalized carrier distribution is exponentially approached with a time constant of $60 \mathrm{fs}$. The maximum of this thermalized distribution is shifted toward lower energies with respect to the excitation pulse spectrum. Then DT contributions at higher energies have to shift over a larger photon energy range $\Delta E$ in a given time $\Delta t$, i.e., $\Delta E / \Delta t$ is larger at higher energies. As shown in Fig. 8(b), this behavior is reflected by the group delay of the optimum pulse shape, for which an increased $\Delta E / \Delta t$ is observed at higher energies. Note that an increased $\Delta E / \Delta t$ corresponds to a decreased slope in the plot of group delay versus photon energy.

With the results from adaptive feedback optical pulseshaping experiments, we gained an intuitive picture of carrier relaxation in the thermalization regime. Data from a semiquantitative model verify these results and give a better understanding of the data. The full quantitative understanding of these data, however, requires a more complex model, ${ }^{16}$ which is beyond the scope of this paper. The experimental key component for gaining insight into the complex light-semiconductor interaction is the adaptive feedback loop. After the loop is optimized as described in Section 3, its high sensitivity allows one to distinguish between spectral phases that lead to only slightly different SI DT signals.

\section{B. Spectrally Resolved Differential Transmission}

In the experiments presented in this subsection, we chose to optimize the DT in narrow spectral windows. We used the phase differences between the pixels of the SLM and the delay between the pump and the probe as genes. In a first experiment, the DT was optimized at a photon energy of $1.625 \mathrm{eV}$ in a spectral window of a $4-\mathrm{meV}$ width. We selected this relatively high photon energy within the pulse spectrum because complex dynamics is observed even with unchirped pulses. As shown in Fig. 10, posi-

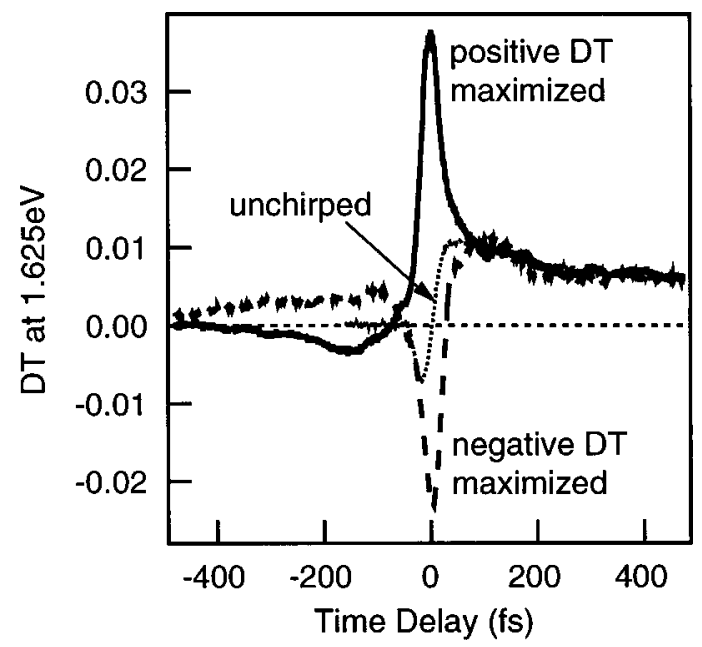

Fig. 10. DT at $1.625 \mathrm{eV}$ for unchirped pulses (dotted curve) and after the positive DT (solid curve) or the negative DT (dashed curve) is maximized. Temperature $300 \mathrm{~K} ; N_{\text {exc }} \approx 3$ $\times 10^{17} \mathrm{~cm}^{-3}$. 
tive and negative DT occur for different time delays. This is the result of the interplay of different DT contributions. For example, negative DT contributions can arise from dynamic Fermi-edge singularity effects. ${ }^{33}$ These effects are limited to early times, at which carrier thermalization has not completely washed out the initially excited carrier distribution. Coherent local-field effects $^{34}$ as well as transient oscillations ${ }^{35}$ can also lead to negative DT. The positive DT contributions can be attributed to phase-space filling. The complex interplay of such contributions provides a well-suited playground for adaptive pulse-shaping schemes.

As shown in Fig. 10, adaptive feedback optical pulse shaping allows one to enhance the positive DT by as much as a factor of approximately 3.5 compared with the DT for unchirped pulses. Alternatively, the feedback loop can maximize the negative DT, which also leads to an enhancement by a factor of more than 3. Hence DT signals of either sign can be enhanced significantly. This means that one can flip the sign of ultrafast semiconductor nonlinearities: The DT signal can be drastically changed from a dominating positive to a dominating negative signal by adjusting the phase of the pump and the probe pulses. After optimization of the positive or the negative DT, the transients exhibit a pronounced ultrafast temporal response with a FWHM of approximately 40-50 fs as shown in Fig. 10. This makes adaptive feedback optical pulse shaping an interesting approach for the optimization of ultrafast all-optical switches. In fact, changing the spectral phase of the pulses allows one to manipulate and to significantly enhance semiconductor nonlinearities on an ultrafast time scale.

Figure 11(a) shows the phase $\varphi_{\mathrm{SR} \text {, max }}$ for the maximization of the positive DT at $1.625 \mathrm{eV}$. Around this photon energy, the optimized spectral phase shows a rapid change of approximately $\pi$. Also shown is the DT spectrum, i.e., the pump-induced transmission change $\Delta T$ normalized to the transmission in absence of the pump $T_{0}$, measured for the optimized phase and the optimized pump-probe delay. Around $1.625 \mathrm{eV}$, a strong enhancement of the DT spectrum can be seen. We note that the strong response at approximately $1.49 \mathrm{eV}$ is due to the excitonic resonance. For the semiconductor continuum nonlinearity, the surprisingly simple shape of the optimized phase and the resonantlike enhancement of the DT spectrum at $1.625 \mathrm{eV}$ remind one of a driven-oscillator behavior. Of course, because of the complex effects involved, ${ }^{33-35}$ this picture can serve only as a qualitative description of the observed amplitude (DT) and phase data. Similar phase shapes and similar resonantly enhanced DT spectra were observed for the maximization of the positive DT at different photon energies. Figure 11(b) shows the optimized phase $\varphi_{-\mathrm{SR} \text {, max }}$ and the corresponding DT spectrum for the maximization of the negative DT at $1.625 \mathrm{eV}$. The negative DT is increased in a relatively narrow spectral range around $1.625 \mathrm{eV}$, and the phase decreases rapidly at higher photon energies.

We also performed experiments in which the positive or negative DT was maximized at $1.625 \mathrm{eV}$ in a broader spectral window (25-meV width). We found that the achievable enhancement factor is significantly reduced compared with the optimization in a $4-\mathrm{meV}$ spectral win- dow. This demonstrates that the DT enhancement is effective only in a small spectral range as indicated by the DT spectra in Fig. 11. Roughly, adaptive feedback optical pulse shaping allows for the spectral focusing of broadband differential transmission signals on an ultrafast time scale.

For the optimization of ultrafast semiconductor nonlinearities in a narrow spectral window, the design of the feedback algorithm, the ES, is again of prime importance. In particular, adequate spectral phase parameters have to be chosen, as explained in Subsection 3.B. For example, a chirp expansion up to the sixth-order dispersion is unable to adequately describe the optimum phase shapes shown in Fig. 11, even though this approach has been used successfully for pulse compression experiments as well as for the maximization of the SI DT. Whereas the independent optimization of the spectral phase differences between all pixels of the SLM gives an enhancement of the DT by more than a factor 3, this enhancement is reduced to a factor of approximately 2 when we use this truncated chirp expansion scheme. We note that the optimized phase shapes for both adequate and inadequate phase descriptions can vary significantly. To explain this result, for the adequate phase description, we consider a global phase optimum and a nonglobal one. If, for the inadequate phase description, the nonglobal phase optimum can be significantly better approximated, the roles of both optima can be reversed. Therefore the algorithm
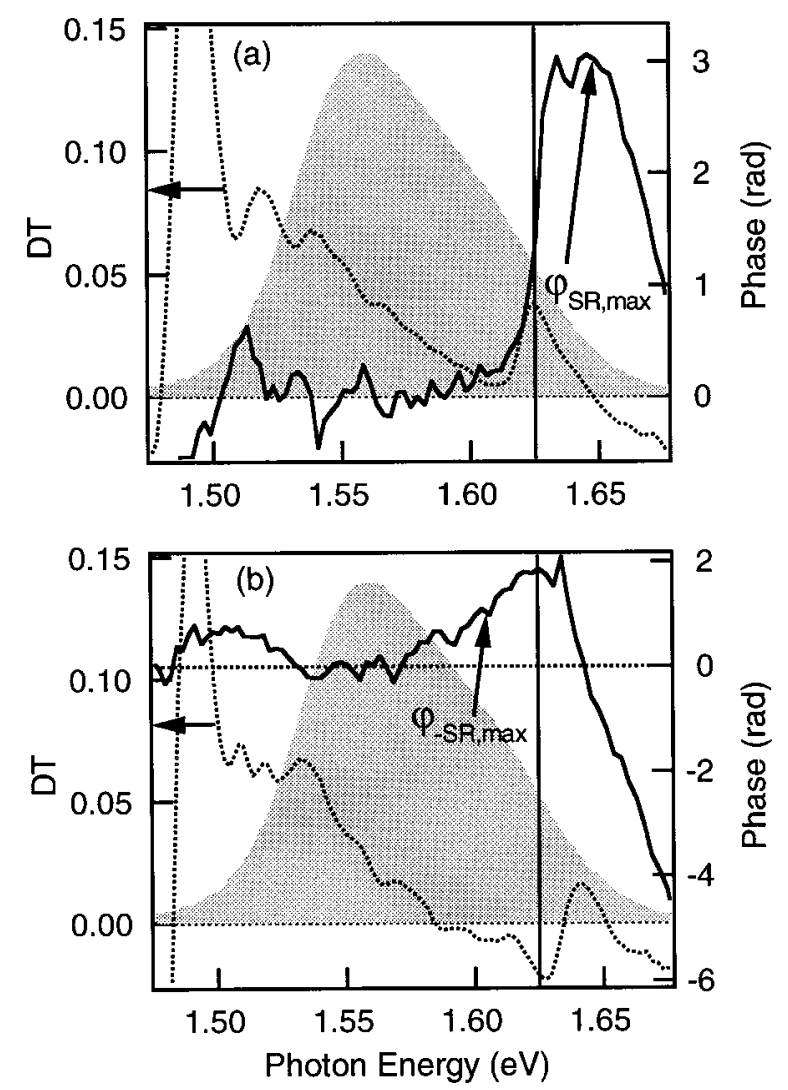

Fig. 11. Optimum phases $\varphi$ (solid curves) that maximize the (a) positive or (b) negative DT (shown in Fig. 10) at $1.625 \mathrm{eV}$. Also shown are the corresponding DT spectra (dotted curves) and the excitation pulse spectrum (shaded area). Temperature $300 \mathrm{~K}$; $N_{\mathrm{exc}} \approx 3 \times 10^{17} \mathrm{~cm}^{-3}$ 
may converge to a completely different phase shape. This underscores that a careful design of the feedback algorithm is important for both an optimum solution to the given control problem and a correct interpretation of the optimized phase shape.

\section{CONCLUSIONS}

We have presented an experimental study of the control of ultrafast semiconductor nonlinearities by adaptive feedback optical pulse shaping. As a computercontrolled algorithm, an evolution strategy directs spectral phase shaping in a feedback loop. We optimized this algorithm, in particular with respect to two issues. First, the spectral phase parameters that are changed by the algorithm are adapted to each control problem. Second, for a given problem and a given set of phase parameters, the crossover operator used by the evolution strategy is optimized in terms of convergence reliability and convergence speed. Following this procedure, an effective algorithm can be designed for other experiments with adaptive feedback optical pulse shaping.

The optimized algorithm proves to be the key element for experiments in which control is exercised over the spectrally integrated differential transmission as well as the differential transmission spectrum. From the optimized phase shape for the maximization of the spectrally integrated differential transmission, an intuitive picture of carrier dynamics in the thermalization regime is developed. Moreover, the differential transmission in a narrow spectral window can be drastically manipulated and significantly enhanced by adaptive feedback optical pulse shaping. This makes the scheme interesting for both semiconductor spectroscopy as well as for the optimization of ultrafast optical switches.

\section{ACKNOWLEDGMENT}

This research has been supported by the Swiss National Science Foundation.

\section{REFERENCES AND NOTES}

1. D. Yelin, D. Meshulach, and Y. Silberberg, "Adaptive femtosecond pulse compression," Opt. Lett. 22, 1793-1795 (1997).

2. T. Baumert, T. Brixner, V. Seyfried, M. Strehle, and G. Gerber, "Femtosecond pulse shaping by an evolutionary algorithm with feedback," Appl. Phys. B 65, 779-782 (1997).

3. A. Efimov, M. D. Moores, N. M. Beach, J. L. Krause, and D. H. Reitze, "Adaptive control of pulse phase in a chirpedpulse amplifier," Opt. Lett. 23, 1915-1917 (1998).

4. E. Zeek, R. Bartels, M. M. Murnane, H. C. Kapteyn, S. Backus, and G. Vdovin, "Adaptive pulse compression for transform-limited 15-fs high-energy pulse generation," Opt. Lett. 25, 587-589 (2000).

5. D. Zeidler, T. Hornung, D. Proch, and M. Motzkus, "Adaptive compression of tunable pulses from a non-collineartype OPA to below 16 fs by feedback-controlled pulse shaping,” Appl. Phys. B 70, S125-S131 (2000).

6. D. Meshulach, D. Yelin, and Y. Silberberg, "Adaptive realtime femtosecond pulse shaping," J. Opt. Soc. Am. B 15, 1615-1619 (1998).

7. F. G. Omenetto, B. P. Luce, and A. J. Taylor, "Genetic algorithm pulse shaping for optimum femtosecond propagation in optical fibers," J. Opt. Soc. Am. B 16, 2005-2009 (1999).
8. R. Bartels, S. Backus, E. Zeek, L. Misoguti, G. Vdovin, I. P. Christov, M. M. Murnane, and H. C. Kapteyn, "Shapedpulse optimization of coherent emission of high-harmonic soft X-rays,” Nature (London) 406, 164-166 (2000).

9. R. S. Judson and H. Rabitz, "Teaching lasers to control molecules," Phys. Rev. Lett. 68, 1500-1503 (1992).

10. C. J. Bardeen, V. V. Yakovlev, K. R. Wilson, S. D. Carpenter, P. M. Weber, and W. S. Warren, "Feedback quantum control of molecular electronic population transfer," Chem. Phys. Lett. 280, 151-158 (1997).

11. A. Assion, T. Baumert, M. Bergt, T. Brixner, B. Kiefer, V. Seyfried, M. Strehle, and G. Gerber, "Control of chemical reactions by feedback-optimized phase-shaped femtosecond laser pulses," Science 282, 919-922 (1998).

12. T. Hornung, R. Meier, D. Zeidler, K.-L. Kompa, D. Proch and M. Motzkus, "Optimal control of one- and two-photon transitions with shaped femtosecond pulses and feedback," Appl. Phys. B 71, 277-284 (2000).

13. T. C. Weinacht, J. Ahn, and P. H. Bucksbaum, "Controlling the shape of a quantum wavefunction," Nature (London) 397, 233-235 (1999).

14. J. Kunde, B. Baumann, S. Arlt, F. Morier-Genoud, U. Siegner, and U. Keller, "Adaptive feedback control of ultrafast semiconductor nonlinearities," Appl. Phys. Lett. 77, 924 926 (2000).

15. J. Shah, Ultrafast Spectroscopy of Semiconductors and Semiconductor Nanostructures, 2nd ed. (Springer-Verlag, Berlin, 1999).

16. H. Haug and A.-P. Jauho, Quantum Kinetics in Transport and Optics of Semiconductors (Springer-Verlag, Berlin, 1996).

17. H. P. Schwefel, Evolution and Optimum Seeking (Wiley, New York, 1995).

18. J. Kunde, S. Arlt, L. Gallmann, F. Morier-Genoud, U. Siegner, and U. Keller, "Sensitive characterization of phase and amplitude semiconductor nonlinearities for broadband $20 \mathrm{fs}$ excitation,” J. Appl. Phys. 88, 1187-1189 (2000).

19. A. M. Weiner, D. E. Leaird, J. S. Patel, and J. R. Wullert, "Programmable femtosecond pulse shaping by use of a multielement liquid-crystal phase modulator," Opt. Lett. 15, 326-328 (1990)

20. M. M. Wefers and K. A. Nelson, "Analysis of programmable ultrashort waveform generation using liquid-crystal spatial light modulators," J. Opt. Soc. Am. B 12, 1343-1362 (1995).

21. M. M. Wefers and K. A. Nelson, "Generation of high-fidelity programmable ultrafast optical waveforms," Opt. Lett. 20, 1047-1049 (1995).

22. M. M. Wefers and K. A. Nelson, "Space-time profiles of shaped ultrafast optical waveforms," IEEE J. Quantum Electron. 32, 161-172 (1996).

23. A. M. Weiner, "Femtosecond pulse shaping using spatial light modulators," Rev. Sci. Instrum. 71, 1929-1960 (2000).

24. A. M. Weiner, D. E. Leaird, J. S. Patel, and J. R. Wullert "Programmable shaping of femtosecond optical pulses by use of 128-element liquid crystal phase modulator," IEEE J. Quantum Electron. 28, 908-920 (1992).

25. R. Trebino, K. W. DeLong, D. N. Fittinghoff, J. Sweetser, M. A. Krumbügel, and B. Richman, "Measuring ultrashort laser pulses in the time-frequency domain using frequencyresolved optical gating," Rev. Sci. Instrum. 68, 1-19 (1997).

26. A. Efimov and D. H. Reitze, "FROG characterization of complex SLM-shaped amplified waveforms," in Conference on Lasers and Electro-Optics, Vol. 39 of 2000 OSA Technical Digest Series (Optical Society of America, Washington D.C., 2000), pp. 621-622.

27. T. Brixner, M. Strehle, and G. Gerber, "Feedback-controlled optimization of amplified femtosecond laser pulses," Appl. Phys. B 68, 281-284 (1999).

28. T. Bäck, Evolutionary Algorithms in Theory and Practice (Oxford U. Press, New York, 1996)

29. The ratio $100 / 15 \approx 7$ is chosen to guarantee a sufficiently low selection pressure. ${ }^{17}$

30. J. Kunde, U. Siegner, S. Arlt, G. Steinmeyer, F. MorierGenoud, and U. Keller, "Potential of femtosecond chirp con- 
trol of ultrabroadband semiconductor continuum nonlinearities," J. Opt. Soc. Am. B 16, 2285-2294 (1999).

31. D. E. Goldberg, Genetic Algorithms in Search, Optimization, and Machine Learning (Addison-Wesley, Reading, Pa., 1993).

32. For the SI DT signal, we took the pump-induced transmission change $\Delta T_{\mathrm{av}}$ normalized to the transmission in absence of the pump $T_{0, \text { av }}$. Both quantities $\Delta T_{\text {av }}$ and $T_{0, \text { av }}$ are averaged individually over the probe spectrum.

33. J.-P. Foing, D. Hulin, M. Joffre, M. K. Jackson, J.-L. Oudar,
C. Tanguay, and M. Combescot, "Absorption edge singularities in highly excited semiconductors," Phys. Rev. Lett. 68, 110-113 (1992).

34. K. El Sayed and C. J. Stanton, "Line-shape analysis of differential transmission spectra in the coherent regime," Phys. Rev. B 55, 9671-9678 (1997).

35. B. Fluegel, N. Peyghambarian, G. Olbright, M. Lindberg, S. W. Koch, M. Joffre, D. Hulin, A. Migus, and A. Antonetti, "Femtosecond studies of coherent transients in semiconductors," Phys. Rev. Lett. 59, 2588-2591 (1987). 